Moroccan J. of Pure and Appl. Anal. (MJPAA)

Volume 4(2), 2018, Pages 111-121

ISSN: Online 2351-8227 - Print 2605-6364

DOI 10.1515/mjpaa-2018-0011

\title{
Opial type inequalities for double Riemann-Stieltjes integrals
}

\author{
HÜSEYIN BUDAK ${ }^{1, a}$
}

Aвstract. In this paper, we establish some Opial type inequalities for Riemann-Stieltjes integrals of functions with two variables. The obtained inequalities generalize those previously demonstrated (see [2]) 2010 Mathematics Subject Classification. Primary: 26D15, 26D10, $26 B 15$.

Key words and phrases. Opial inequality, Hölder's inequality.

\section{Introduction}

Opial's inequality and its generalizations, extensions and discretizations, play a fundamental role in establishing the existence and uniqueness of initial and boundary value problems for ordinary and partial differential equations as well as difference equations.

In 1960, Z. Opial established the following interesting integral inequality which is known as Opial inequality in the literature[12]:

Theorem 1.1. Let $x(t) \in C^{(1)}[0, h]$ be such that $x(0)=x(h)=0$, and $x(t)>0$ in $(0, h)$. Then, the following inequality holds

$$
\int_{0}^{h}\left|x(t) x^{\prime}(t)\right| d t \leq \frac{h}{4} \int_{0}^{h}\left(x^{\prime}(t)\right)^{2} d t
$$

The constant $h / 4$ is the best possible

Over the years, a large number of papers have been appeared in the literature which deals various generalizations, as well as discrete form of Opial inequality and its generalizations, for some of them see [4], [5], [8], [9], [13]-[16], [26]-[28].

On the other hand, G. S. Yang gave the following generalization (1.1) for the function of two variables [29]

Received February 27, 2019 - Accepted April 7, 2019.

(C) The Author(s) 2017. This article is published with open access by Sidi Mohamed Ben Abdallah University.

This work was supported by Scientific Research Projects Coordination Unit of Düzce University. Project number 2019.05.04.902.

${ }^{1}$ Department of Mathematics, Faculty of Science and Arts, Düzce University, Düzce, Turkey

a e-mail:mhsyn.budak@gmail.com . 
Theorem 1.2. If $f(t, s), f_{1}(t, s)$ and $f_{12}(t, s)$ are continuous functions on $[a, b] \times[c, d]$ and if $f(a, s)=f(b, s)=f_{1}(t, c)=$ $f_{1}(t, d)=0$ for $a \leq t \leq b, c \leq s \leq d$, then

$$
\int_{a}^{b} \int_{c}^{d}|f(t, s)|\left|f_{12}(t, s)\right| d s d t \leq \frac{(b-a)(d-c)}{8} \int_{a}^{b} \int_{c}^{d}\left|f_{12}(t, s)\right|^{2} d s d t
$$

where

$$
f_{1}(t, s)=\frac{\partial}{\partial t} f(t, s) \text { and } f_{12}(s, t)=\frac{\partial^{2}}{\partial t \partial s} f(t, s) .
$$

Over the years, many articles are dedicated to the generalizations of inequality (1.2). B. G. Pachpatte published some papers which focus on the generalizations of the inequality (1.2). For some of these generalizations, see [17][22]. Moreover using two functions and their partial derivatives, W. S. Cheung established some generalizations of the inequality (1.2) in [6]. For the other Opial type inequalities in higher dimension, see [1]-[3], [7], [23]-[25].

The following Lemma proved by Moricz in [11] is usefull to obtain our main result:

Lemma 1.1. (Integrating by parts) If $f(t, s)$ is continuous on renctangle $Q=[a, b] \times[c, d]$ and $\alpha(t, s) \in B V_{H}(Q)$, then $\alpha(t, s)$ is integrable with respect to $f(t, s)$ over $Q$ in the Riemann-Stieltjes sense, and

$$
\begin{aligned}
\int_{a}^{b} \int_{c}^{d} f(t, s) d_{t} d_{s} \alpha(t, s)= & \int_{a}^{b} \int_{c}^{d} \alpha(t, s) d_{t} d_{s} f(t, s) \\
& -\int_{a}^{b} \alpha(t, d) d_{t} f(t, d)+\int_{a}^{b} \alpha(t, c) d_{t} f(t, c) \\
& -\int_{c}^{d} \alpha(b, s) d_{s} f(b, s)+\int_{c}^{d} \alpha(a, s) d_{s} f(a, s) \\
= & f(b, d) \alpha(b, d)-f(b, c) \alpha(b, c)-f(a, d) \alpha(a, d)+f(a, c) \alpha(a, c) .
\end{aligned}
$$

\section{Generalized Opial type inequalities}

In this section, we establish some Opial type inequalities for double Riemann-Stieltjes integrals.

Theorem 2.1. Let $f(s, t), f_{1}(s, t), f_{12}(s, t), g(s, t), g_{1}(s, t), g_{12}(s, t)$ and $\alpha(x, y)$ be continuous on $\Delta:=[a, b] \times[c, d]$ and let $f(x, y)$ and $g(x, y)$ be integrable with respect to $\alpha(x, y)$ over $\Delta$ in the Riemann-Stieltjes sense. If $g(a, s)=g(b, s)=$ $g_{1}(t, c)=g_{1}(t, d)=0$ for $(t, s) \in \Delta$, then for all $(x, y) \in \Delta$ we have

$$
\begin{aligned}
& \int_{a}^{b} \int_{c}^{d}\left|f_{12}(t, s) g(t, s)\right| d_{t} d_{s} \alpha(t, s) \\
\leq & \frac{1}{4}\left[(b-a) \int_{a}^{b} \int_{c}^{d} Q(s, y)\left|f_{12}(t, s)\right|^{2} d_{t} d_{s} \alpha(t, s)+(d-c) \int_{a}^{b} \int_{c}^{d} P(t, x)\left|f_{12}(t, s)\right|^{2} d_{t} d_{s} \alpha(t, s)\right]^{\frac{1}{2}} \\
\times & {\left[\int_{a}^{b} \int_{c}^{d}|\alpha(b, y)-\alpha(a, y)-\alpha(b, s)+\alpha(a, s)|\left|g_{12}(t, s)\right|^{2} d s d t\right.} \\
+ & \left.\int_{a}^{b} \int_{c}^{d}|\alpha(t, d)-\alpha(x, d)-\alpha(t, c)+\alpha(x, c)|\left|g_{12}(t, s)\right|^{2} d s d t\right]^{\frac{1}{2}}
\end{aligned}
$$


OPIAL TYPE INEQUALITIES FOR DOUBLE RIEMANN-STIELTJES INTEGRALS

$$
\begin{aligned}
\leq & \frac{1}{8}\left[(b-a) \int_{a}^{b} \int_{c}^{d} Q(s, y)\left|f_{12}(t, s)\right|^{2} d_{t} d_{s} \alpha(t, s)+(d-c) \int_{a}^{b} \int_{c}^{d} P(t, x)\left|f_{12}(t, s)\right|^{2} d_{t} d_{s} \alpha(t, s)\right. \\
& +\int_{a}^{b} \int_{c}^{d}|\alpha(b, y)-\alpha(a, y)-\alpha(b, s)+\alpha(a, s)|\left|g_{12}(t, s)\right|^{2} d s d t \\
& \left.+\int_{a}^{b} \int_{c}^{d}|\alpha(t, d)-\alpha(x, d)-\alpha(t, c)+\alpha(x, c)|\left|g_{12}(t, s)\right|^{2} d s d t\right]
\end{aligned}
$$

where

$$
P(t, x)=\left\{\begin{array}{ll}
t-a, & a \leq t \leq x \\
b-t, & x \leq t \leq b
\end{array} \quad \text { and } \quad Q(s, y)= \begin{cases}s-c, & c \leq s \leq y \\
d-s, & y \leq s \leq d .\end{cases}\right.
$$

Proof. In order to prove Theorem 2.1, we consider the following four cases:

Case I: Let $g(a, s)=g_{1}(t, c)=0$ for $(t, s) \in[a, b] \times[c, d]$.

Since $g(a, s)=g_{1}(t, c)=0$, we can write

$$
g(t, s)=\int_{a}^{t} \int_{c}^{s} g_{12}(u, v) d u d v
$$

then we have

$$
\begin{aligned}
& \int_{a}^{b} \int_{c}^{d}\left|f_{12}(t, s) g(t, s)\right| d_{t} d_{s} \alpha(t, s) \\
= & \int_{a}^{b} \int_{c}^{d}(t-a)^{\frac{1}{2}}(s-c)^{\frac{1}{2}}\left|f_{12}(t, s)\right|(t-a)^{-\frac{1}{2}}(s-c)^{-\frac{1}{2}}|g(t, s)| d_{t} d_{s} \alpha(t, s) \\
= & \int_{a}^{b} \int_{c}^{d}(t-a)^{\frac{1}{2}}(s-c)^{\frac{1}{2}}\left|f_{12}(t, s)\right|(t-a)^{-\frac{1}{2}}(s-c)^{-\frac{1}{2}}\left|\int_{a}^{t} \int_{c}^{s} g_{12}(u, v) d u d v\right| d_{t} d_{s} \alpha(t, s) .
\end{aligned}
$$

By using Cauchy-Schwarz inequality for Riemann integrals, we get

$$
\begin{aligned}
& (t-a)^{-\frac{1}{2}}(s-c)^{-\frac{1}{2}}\left|\int_{a}^{t} \int_{c}^{s} g_{12}(u, v) d u d v\right| \\
\leq & (t-a)^{-\frac{1}{2}}(s-c)^{-\frac{1}{2}}\left(\int_{a}^{t} \int_{c}^{s} d u d v\right)^{\frac{1}{2}}\left(\int_{a}^{t} \int_{c}^{s}\left|g_{12}(u, v)\right|^{2} d u d v\right)^{\frac{1}{2}} \\
= & \left(\int_{a}^{t} \int_{c}^{s}\left|g_{12}(u, v)\right|^{2} d u d v\right)^{\frac{1}{2}} .
\end{aligned}
$$

Substituting the inequality (2.3) in (2.2), we obtain

$$
\int_{a}^{b} \int_{c}^{d}\left|f_{12}(t, s) g(t, s)\right| d_{t} d_{s} \alpha(t, s)
$$




$$
\leq \int_{a}^{b} \int_{c}^{d}(t-a)^{\frac{1}{2}}(s-c)^{\frac{1}{2}}\left|f_{12}(t, s)\right|\left(\int_{a}^{t} \int_{c}^{s}\left|g_{12}(u, v)\right|^{2} d u d v\right)^{\frac{1}{2}} d_{t} d_{s} \alpha(t, s) .
$$

By using Cauchy-Schwarz inequality for Riemann-Stieltjes integrals, we obtain

$$
\begin{aligned}
& \int_{a}^{b} \int_{c}^{d}\left|f_{12}(t, s) g(t, s)\right| d_{t} d_{s} \alpha(t, s) \\
\leq & \left(\int_{a}^{b} \int_{c}^{d}(t-a)(s-c)\left|f_{12}(t, s)\right|^{2} d_{t} d_{s} \alpha(t, s)\right)^{\frac{1}{2}}\left(\int_{a}^{b} \int_{c}^{d}\left(\int_{a}^{t} \int_{c}^{s}\left|g_{12}(u, v)\right|^{2} d u d v\right) d_{t} d_{s} \alpha(t, s)\right)^{\frac{1}{2}} .
\end{aligned}
$$

By the integration by parts for Riemann-Stieltjes integrals (Lemma 1.1), we establish

$$
\begin{aligned}
& \int_{a}^{b} \int_{c}^{d}\left(\int_{a}^{t} \int_{c}^{s}\left|g_{12}(u, v)\right|^{2} d u d v\right) d_{t} d_{s} \alpha(t, s) \\
= & -\int_{a}^{b} \int_{c}^{d} \alpha(t, d)\left|g_{12}(t, v)\right|^{2} d v d t-\int_{a}^{b} \int_{c}^{d} \alpha(b, s)\left|g_{12}(u, s)\right|^{2} d s d u \\
+ & \alpha(b, d) \int_{a}^{b} \int_{c}^{d}\left|g_{12}(u, v)\right|^{2} d v d u+\int_{a}^{b} \int_{c}^{d} \alpha(t, s)\left|g_{12}(t, s)\right|^{2} d s d t \\
= & \int_{a}^{b} \int_{c}^{d}[-\alpha(t, d)-\alpha(b, s)+\alpha(b, d)+\alpha(t, s)]\left|g_{12}(t, v)\right|^{2} d s d t .
\end{aligned}
$$

By the using the equality (2.6) in (2.4), we obtain the following inequality

$$
\begin{aligned}
& \int_{a}^{b} \int_{c}^{d}\left|f_{12}(t, s) g(t, s)\right| d_{t} d_{s} \alpha(t, s) \\
\leq & \left(\int_{a}^{b} \int_{c}^{d}(t-a)(s-c)\left|f_{12}(t, s)\right|^{2} d_{t} d_{s} \alpha(t, s)\right)^{\frac{1}{2}} \\
& \times\left(\int_{a}^{b} \int_{c}^{d}[-\alpha(t, d)-\alpha(b, s)+\alpha(b, d)+\alpha(t, s)]\left|g_{12}(t, v)\right|^{2} d s d t\right)^{\frac{1}{2}} .
\end{aligned}
$$

Case II: Let $g(a, s)=g_{1}(t, d)=0$ for $(t, s) \in[a, b] \times[c, d]$.

We get

$$
g(t, s)=-\int_{a}^{t} \int_{s}^{d} g_{12}(u, v) d u d v
$$

for $(t, s) \in[a, b] \times[c, d]$. Then by Cauchy-Schwarz inequality for Riemann integrals, it follows that

$$
\int_{a}^{b} \int_{c}^{d}\left|f_{12}(t, s) g(t, s)\right| d_{t} d_{s} \alpha(t, s)
$$


OPIAL TYPE INEQUALITIES FOR DOUBLE RIEMANN-STIELTJES INTEGRALS

$$
\begin{aligned}
& =\int_{a}^{b} \int_{c}^{d}(t-a)^{\frac{1}{2}}(d-s)^{\frac{1}{2}}\left|f_{12}(t, s)\right|(t-a)^{-\frac{1}{2}}(d-s)^{-\frac{1}{2}}\left|\int_{a}^{t} \int_{s}^{d} g_{12}(u, v) d u d v\right| d_{t} d_{s} \alpha(t, s) \\
& \leq \int_{a}^{b} \int_{c}^{d}(t-a)^{\frac{1}{2}}(d-s)^{\frac{1}{2}}\left|f_{12}(t, s)\right|\left(\int_{a}^{b} \int_{c}^{d}\left|g_{12}(u, v)\right|^{2} d u d v\right)^{\frac{1}{2}} d_{t} d_{s} \alpha(t, s) .
\end{aligned}
$$

By applying Cauchy-Schwarz inequality for Riemann-Stieltjes integrals, we get

$$
\begin{aligned}
& \int_{a}^{b} \int_{c}^{d}\left|f_{12}(t, s) g(t, s)\right| d_{t} d_{s} \alpha(t, s) \\
\leq & \left(\int_{a}^{b} \int_{c}^{d}(t-a)(d-s)\left|f_{12}(t, s)\right|^{2} d_{t} d_{s} \alpha(t, s)\right)^{\frac{1}{2}}\left(\int_{a}^{b} \int_{c}^{d}\left(\int_{a}^{b} \int_{c}^{d}\left|g_{12}(u, v)\right|^{2} d u d v\right) d_{t} d_{s} \alpha(t, s)\right)^{\frac{1}{2}} .
\end{aligned}
$$

By integration by parts for Riemann-Stieltjes integrals, one can show that

$$
\begin{aligned}
& \int_{a}^{b} \int_{c}^{d}\left(\int_{a}^{b} \int_{c}^{d}\left|g_{12}(u, v)\right|^{2} d u d v\right) d_{t} d_{s} \alpha(t, s) \\
= & \int_{a}^{b} \int_{c}^{d}[\alpha(t, c)+\alpha(b, s)-\alpha(b, c)-\alpha(t, s)]\left|g_{12}(u, v)\right|^{2} d s d t .
\end{aligned}
$$

Thus, we have

$$
\begin{aligned}
& \int_{a}^{b} \int_{c}^{d}\left|f_{12}(t, s) g(t, s)\right| d_{t} d_{s} \alpha(t, s) \\
\leq & \left(\int_{a}^{b} \int_{c}^{d}(t-a)(d-s)\left|f_{12}(t, s)\right|^{2} d_{t} d_{s} \alpha(t, s)\right)^{\frac{1}{2}} \\
& \times\left(\int_{a}^{b} \int_{c}^{d}[\alpha(t, c)+\alpha(b, s)-\alpha(b, c)-\alpha(t, s)]\left|g_{12}(u, v)\right|^{2} d s d t\right)^{\frac{1}{2}} .
\end{aligned}
$$

Case III: Let $g(b, s)=g_{1}(t, c)=0$ for $(t, s) \in[a, b] \times[c, d]$. Then we have

$$
g(t, s)=-\int_{t}^{b} \int_{c}^{s} g_{12}(u, v) d u d v .
$$

Case IV: Let $g(b, s)=g_{1}(t, d)=0$ for $(t, s) \in[a, b] \times[c, d]$. we can write

$$
g(t, s)=\int_{t}^{b} \int_{s}^{d} g_{12}(u, v) d u d v .
$$


By following similar to those in proof of (2.7) and (2.8), but with suitable modifications, we establish the following inequalities in Case III and Case IV:

$$
\begin{aligned}
& \int_{a}^{b} \int_{c}^{d}\left|f_{12}(t, s) g(t, s)\right| d_{t} d_{s} \alpha(t, s) \\
\leq & \left(\int_{a}^{b} \int_{c}^{d}(b-t)(s-c)\left|f_{12}(t, s)\right|^{2} d_{t} d_{s} \alpha(t, s)\right)^{\frac{1}{2}} \\
& \times\left(\int_{a}^{b} \int_{c}^{d}[\alpha(a, s)+\alpha(t, d)-\alpha(a, d)-\alpha(t, s)]\left|g_{12}(t, s)\right|^{2} d s d t\right)^{\frac{1}{2}}, \\
& \int_{a}^{b} \int_{c}^{d}\left|f_{12}(t, s) g(t, s)\right| d_{t} d_{s} \alpha(t, s) \\
\leq & \left(\int_{a}^{b} \int_{c}^{d}(b-t)(d-s)\left|f_{12}(t, s)\right|^{2} d_{t} d_{s} \alpha(t, s)\right)^{\frac{1}{2}} \\
& \times\left(\int_{a}^{b} \int_{c}^{d}[-\alpha(t, c)-\alpha(a, s)+\alpha(a, c)+\alpha(t, s)]\left|g_{12}(t, s)\right|^{2} d s d t\right)^{\frac{1}{2}},
\end{aligned}
$$

respectively.

Since $g(a, s)=g_{1}(t, c)=0$ for $(t, s) \in[a, b] \times[c, d]$, if we write the inequality (2.7) for the rectangles $[a, b] \times[c, y]$ and $[a, x] \times[c, d]$ for $(x, y) \in[a, b] \times[c, d]$, then we have

$$
\begin{aligned}
& \int_{a}^{b} \int_{c}^{y}\left|f_{12}(t, s) g(t, s)\right| d_{t} d_{s} \alpha(t, s) \\
\leq & \left(\int_{a}^{b} \int_{c}^{y}(t-a)(s-c)\left|f_{12}(t, s)\right|^{2} d_{t} d_{s} \alpha(t, s)\right)^{\frac{1}{2}} \\
& \times\left(\int_{a}^{b} \int_{c}^{y}[-\alpha(t, y)-\alpha(b, s)+\alpha(b, y)+\alpha(t, s)]\left|g_{12}(t, v)\right|^{2} d s d t\right)^{\frac{1}{2}} .
\end{aligned}
$$

and

$$
\begin{gathered}
\int_{a}^{x} \int_{c}^{d}\left|f_{12}(t, s) g(t, s)\right| d_{t} d_{s} \alpha(t, s) \\
\leq\left(\int_{a}^{x} \int_{c}^{d}(t-a)(s-c)\left|f_{12}(t, s)\right|^{2} d_{t} d_{s} \alpha(t, s)\right)^{\frac{1}{2}}
\end{gathered}
$$




$$
\times\left(\int_{a}^{x} \int_{c}^{d}[-\alpha(t, d)-\alpha(x, s)+\alpha(x, d)+\alpha(t, s)]\left|g_{12}(t, v)\right|^{2} d s d t\right)^{\frac{1}{2}} .
$$

respectively.

As $g(a, s)=g_{1}(t, d)=0$ for $(t, s) \in[a, b] \times[c, d]$, if we apply the inequality (2.8) for the rectangles $[a, b] \times[y, d]$ and $[a, x] \times[c, d]$ for $(x, y) \in[a, b] \times[c, d]$, then we get

$$
\begin{aligned}
& \int_{a}^{b} \int_{y}^{d}\left|f_{12}(t, s) g(t, s)\right| d_{t} d_{s} \alpha(t, s) \\
\leq & \left(\int_{a}^{b} \int_{y}^{d}(t-a)(d-s)\left|f_{12}(t, s)\right|^{2} d_{t} d_{s} \alpha(t, s)\right)^{\frac{1}{2}} \\
& \times\left(\int_{a}^{b} \int_{y}^{d}[\alpha(t, y)+\alpha(b, s)-\alpha(b, y)-\alpha(t, s)]\left|g_{12}(u, v)\right|^{2} d s d t\right)^{\frac{1}{2}} .
\end{aligned}
$$

and

$$
\begin{aligned}
& \int_{a}^{x} \int_{c}^{d}\left|f_{12}(t, s) g(t, s)\right| d_{t} d_{s} \alpha(t, s) \\
\leq & \left(\int_{a}^{x} \int_{c}^{d}(t-a)(d-s)\left|f_{12}(t, s)\right|^{2} d_{t} d_{s} \alpha(t, s)\right)^{\frac{1}{2}} \\
& \times\left(\int_{a}^{x} \int_{c}^{d}[\alpha(t, c)+\alpha(b, s)-\alpha(b, c)-\alpha(t, s)]\left|g_{12}(u, v)\right|^{2} d s d t\right)^{\frac{1}{2}} .
\end{aligned}
$$

Similarly, since $g(b, s)=g_{1}(t, c)=0$ for $(t, s) \in[a, b] \times[c, d]$, if we write the inequality (2.9) for the rectangles $[a, b] \times[c, y]$ and $[x, b] \times[c, d]$ for $(x, y) \in[a, b] \times[c, d]$, then we have

$$
\begin{aligned}
& \int_{a}^{b} \int_{c}^{y}\left|f_{12}(t, s) g(t, s)\right| d_{t} d_{s} \alpha(t, s) \\
\leq & \left(\int_{a}^{b} \int_{c}^{y}(b-t)(s-c)\left|f_{12}(t, s)\right|^{2} d_{t} d_{s} \alpha(t, s)\right)^{\frac{1}{2}} \\
& \times\left(\int_{a}^{b} \int_{c}^{y}[\alpha(a, s)+\alpha(t, y)-\alpha(a, y)-\alpha(t, s)]\left|g_{12}(t, s)\right|^{2} d s d t\right)^{\frac{1}{2}},
\end{aligned}
$$

and

$$
\int_{x}^{b} \int_{c}^{d}\left|f_{12}(t, s) g(t, s)\right| d_{t} d_{s} \alpha(t, s)
$$




$$
\begin{aligned}
\leq & \left(\int_{x}^{b} \int_{c}^{d}(b-t)(s-c)\left|f_{12}(t, s)\right|^{2} d_{t} d_{s} \alpha(t, s)\right)^{\frac{1}{2}} \\
& \times\left(\int_{x}^{b} \int_{c}^{d}[\alpha(x, s)+\alpha(t, d)-\alpha(x, d)-\alpha(t, s)]\left|g_{12}(t, s)\right|^{2} d s d t\right)^{\frac{1}{2}},
\end{aligned}
$$

Finally, as $g(b, s)=g_{1}(t, d)=0$ for $(t, s) \in[a, b] \times[c, d]$, if we apply the inequality (2.10) for the rectangles $[a, b] \times[y, d]$ and $[x, b] \times[c, d]$ for $(x, y) \in[a, b] \times[c, d]$, then we have

$$
\begin{aligned}
& \int_{a}^{b} \int_{y}^{d}\left|f_{12}(t, s) g(t, s)\right| d_{t} d_{s} \alpha(t, s) \\
\leq & \left(\int_{a}^{b} \int_{y}^{d}(b-t)(d-s)\left|f_{12}(t, s)\right|^{2} d_{t} d_{s} \alpha(t, s)\right)^{\frac{1}{2}} \\
& \times\left(\int_{a}^{b} \int_{y}^{d}[-\alpha(t, y)-\alpha(a, s)+\alpha(a, y)+\alpha(t, s)]\left|g_{12}(t, s)\right|^{2} d s d t\right)^{\frac{1}{2}},
\end{aligned}
$$

and

$$
\begin{aligned}
& \int_{x}^{b} \int_{c}^{d}\left|f_{12}(t, s) g(t, s)\right| d_{t} d_{s} \alpha(t, s) \\
\leq & \left(\int_{x}^{b} \int_{c}^{d}(b-t)(d-s)\left|f_{12}(t, s)\right|^{2} d_{t} d_{s} \alpha(t, s)\right)^{\frac{1}{2}} \\
& \times\left(\int_{x}^{b} \int_{c}^{d}[-\alpha(t, c)-\alpha(x, s)+\alpha(x, c)+\alpha(t, s)]\left|g_{12}(t, s)\right|^{2} d s d t\right)^{\frac{1}{2}},
\end{aligned}
$$

Let $a_{1}, a_{2}, \ldots, a_{n}$ and $b_{1}, b_{2}, \ldots, b_{n}$ be reel numbers. Then we have the following Cauchy-Scwarz inequality

$$
a_{1} b_{1}+a_{2} b_{2}+\ldots+a_{n} b_{n} \leq\left(a_{1}^{2}+a_{2}^{2}+\ldots .+a_{n}^{2}\right)^{\frac{1}{2}}\left(b_{1}^{2}+b_{2}^{2}+\ldots .+b_{n}^{2}\right)^{\frac{1}{2}} .
$$

If we add the inequalities (2.11)-(2.18), then by using the Cauchy-Schwarz inequality (2.19), we obtain

$$
\begin{aligned}
& 4 \int_{a}^{b} \int_{c}^{d}\left|f_{12}(t, s) g(t, s)\right| d_{t} d_{s} \alpha(t, s) \\
\leq & {\left[\int_{a}^{b} \int_{c}^{y}(b-a)(s-c)\left|f_{12}(t, s)\right|^{2} d_{t} d_{s} \alpha(t, s)+\int_{a}^{x} \int_{c}^{d}(t-a)(d-c)\left|f_{12}(t, s)\right|^{2} d_{t} d_{s} \alpha(t, s)\right.} \\
& \left.+\int_{a}^{b} \int_{y}^{d}(b-a)(d-s)\left|f_{12}(t, s)\right|^{2} d_{t} d_{s} \alpha(t, s)+\int_{x}^{b} \int_{c}^{d}(b-t)(d-c)\left|f_{12}(t, s)\right|^{2} d_{t} d_{s} \alpha(t, s)\right]^{\frac{1}{2}}
\end{aligned}
$$


OPIAL TYPE INEQUALITIES FOR DOUBLE RIEMANN-STIELTJES INTEGRALS

$$
\begin{aligned}
& \times\left[\int_{a}^{b} \int_{c}^{y}[\alpha(b, y)-\alpha(a, y)-\alpha(b, s)+\alpha(a, s)]\left|g_{12}(t, s)\right|^{2} d s d t\right. \\
& +\int_{a}^{x} \int_{c}^{d}[\alpha(t, c)-\alpha(x, c)-\alpha(t, d)+\alpha(x, d)]\left|g_{12}(t, s)\right|^{2} d s d t \\
& +\int_{a}^{b} \int_{y}^{d}[\alpha(b, s)-\alpha(b, y)-\alpha(a, s)+\alpha(a, y)]\left|g_{12}(t, s)\right|^{2} d s d t \\
& \left.+\int_{x}^{b} \int_{c}^{d}[\alpha(t, d)-\alpha(x, d)-\alpha(t, c)+\alpha(x, c)]\left|g_{12}(t, s)\right|^{2} d s d t\right]^{\frac{1}{2}} \\
& =\left[(b-a) \int_{a}^{b} \int_{c}^{d} Q(s, y)\left|f_{12}(t, s)\right|^{2} d_{t} d_{s} \alpha(t, s)+(d-c) \int_{a}^{b} \int_{c}^{d} P(t, x)\left|f_{12}(t, s)\right|^{2} d_{t} d_{s} \alpha(t, s)\right]^{\frac{1}{2}} \\
& \times\left[\int_{a}^{b} \int_{c}^{d}|\alpha(b, y)-\alpha(a, y)-\alpha(b, s)+\alpha(a, s)|\left|g_{12}(t, s)\right|^{2} d s d t+\right. \\
& \left.+\int_{a}^{b} \int_{c}^{d}|\alpha(t, d)-\alpha(x, d)-\alpha(t, c)+\alpha(x, c)|\left|g_{12}(t, s)\right|^{2} d s d t\right]^{\frac{1}{2}} \text {. }
\end{aligned}
$$

This proves the first inequality in (2.1).

The proof of the second inequality in (2.1) is obvious from the fact that $\sqrt{p q} \leq \frac{1}{2}(p+q)$, for $p, q>0$.

Corollary 2.1. Assume that assumptions of Theorem 2.1 hold. If $w:[a, b] \times[c, d] \rightarrow[0, \infty)$ is continuous and we chose $\alpha(t, s)=\int_{a}^{t} \int_{c}^{t} w(u, v) d v d u$, then we obtain the following weighted inequalities

$$
\begin{aligned}
& \int_{a}^{b} \int_{c}^{d}\left|f_{12}(t, s) g(t, s)\right| w(t, s) d s d t \\
\leq & \left.\frac{1}{4}\left[(b-a) \int_{a}^{b} \int_{c}^{d} Q(s, y)\left|f_{12}(t, s)\right|^{2} w(t, s) d s d t+(d-c) \int_{a}^{b} \int_{c}^{d} P(t, x)\left|f_{12}(t, s)\right|^{2} w(t, s) d s d t\right]\right]^{\frac{1}{2}} \\
\times & {\left[\int_{a}^{b} \int_{c}^{d}\left(\left|\int_{a}^{b} \int_{s}^{y} w(u, v) d v d u\right|+\left|\int_{t}^{x} \int_{c}^{d} w(u, v) d v d u\right|\right)\left|g_{12}(t, s)\right|^{2} d s d t\right]^{\frac{1}{2}} } \\
\leq & {\left[(b-a) \int_{a}^{b} \int_{c}^{d} Q(s, y)\left|f_{12}(t, s)\right|^{2} w(t, s) d s d t+(d-c) \int_{a}^{b} \int_{c}^{d} P(t, x)\left|f_{12}(t, s)\right|^{2} w(t, s) d s d t\right.} \\
+ & \left.\int_{a}^{b} \int_{c}^{d}\left(\left|\int_{a}^{b} \int_{s}^{y} w(u, v) d v d u\right|+\left|\int_{t}^{x} \int_{c}^{d} w(u, v) d v d u\right|\right)\left|g_{12}(t, s)\right|^{2} d s d t\right]
\end{aligned}
$$

where $Q(s, y)$ and $P(t, x)$ are defined as in Theorem 2.1. 
Remark 2.1. If we choose $w(t, s)=1$ for all $(t, s) \in[a, b] \times[c, d]$ in Corollary 2.1, then we have the following inequalities

$$
\begin{aligned}
& \int_{a}^{b} \int_{c}^{d}\left|f_{12}(t, s) g(t, s)\right| d s d t \\
\leq & \frac{1}{4}\left[(b-a) \int_{a}^{b} \int_{c}^{d} Q(s, y)\left|f_{12}(t, s)\right|^{2} d s d t+(d-c) \int_{a}^{b} \int_{c}^{d} P(t, x)\left|f_{12}(t, s)\right|^{2} d s d t\right]^{\frac{1}{2}} \\
\times & {\left[\int_{a}^{b} \int_{c}^{d}((b-a)|y-s|+(d-c)|x-t|)\left|g_{12}(t, s)\right|^{2} d s d t\right]^{\frac{1}{2}} } \\
\leq & \frac{1}{8}\left[(b-a) \int_{a}^{b} \int_{c}^{d} Q(s, y)\left|f_{12}(t, s)\right|^{2} d s d t+(d-c) \int_{a}^{b} \int_{c}^{d} P(t, x)\left|f_{12}(t, s)\right|^{2} d s d t\right. \\
+ & \left.\int_{a}^{b} \int_{c}^{d}((b-a)|y-s|+(d-c)|x-t|)\left|g_{12}(t, s)\right|^{2} d s d t t\right]
\end{aligned}
$$

which is proved by Budak and Sarkkaya in [2].

\section{Concluding Remarks}

In this study, we established the generalized Opial inequality for Riemann-Stieltjes integrals of functions with two independent variables. In further studies, one can obtain weighted version of the inequality given this paper.

\section{References}

[1] R.P. Agarwal and P. Y. H. Pang,: Sharp opial-type inequalities in two variables. Appl Anal. 56(3):227-242 (1996).

[2] H. Budak and Sarikaya, Refinements of Opial type inequalities in two variables, ResearchGate Article: www.researchgate.net/publication/329091454.

[3] Z. Changjian, and W. Cheung,On improvements of Opial-type inequalities. Georgian Mathematical Journal, 21(4), pp. 415-419, 2014.

[4] W.S. Cheung, Some new Opial-type inequalities, Mathematika, 37 (1990), 136-142.

[5] W.S. Cheung, Some generalized Opial-type inequalities, J. Math. Anal. Appl., 162 (1991), 317- 321.

[6] W.S. Cheung, On Opial-type inequalities in two variables, Aequationes Mathematicae 38 (1989) 236-244.

[7] W.S. Cheung, Opial-type inequalities with $\mathrm{m}$ functions in $\mathrm{n}$ variables. Mathematika, 39(2), 319-326, 1992.

[8] S. S. Dragomir, Generalizations of Opial's inequalities for two functions and applications, Preprint RGMIA Res. Rep. Coll. 21 (2018), Art. 64.

[9] S. S. Dragomir,Generalizations of Opial's inequalities for Riemann-Stieltjes integrals with applications, Preprint RGMIA Res. Rep. Coll. 21 (2018), Art. 69.

[10] C. T. Lin and G. S.Yang, A generalized Opial's inequality in two variables. Tamkang J. Math. 15 (1984), $115-122$.

[11] F. Moricz, Order of magnitude of double Fourier coefficients of functions of bounded variation, Analysis (Munich), 22(4), 2002, 335-345.

[12] Z. Opial, Sur une inegaliti, Ann. Polon. Math. 8 (1960), 29-32.

[13] B. G. Pachpatte, On Opial-type integral inequalities , J. Math. Anal. Appl. 120 (1986), 547-556.

[14] B. G. Pachpatte, Some inequalities similar to Opial's inequality , Demonstratio Math. 26 (1993), 643-647.

[15] B. G. Pachpatte, A note on some new Opial type integral inequalities, Octogon Math. Mag. 7 (1999), 80-84. 
[16] B. G. Pachpatte, On some inequalities of the Weyl type, An. Stiint. Univ. “Al.I. Cuza" Iasi 40 (1994), $89-95$.

[17] B. G. Pachpatte. On Opial type integral inequalities, J. Math. Analy. Appl., 120, 547-556 (1986).

[18] B. G. Pachpatte, On two inequalities similar to Opial's inequality in two independent variables, Periodica Math. Hungarica 18, 137-141, 1987.

[19] B. G. Pachpatte, On an inequality of opial type in two variables, Indian J. Pure Appl. Math., 23(9), 657-661, 1992.

[20] B.G. Pachpatte, On two independent variable Opial-type integral inequalities, J. Math. Anal. Appl. 125(1987), 47-57.

[21] B.G. Pachpatte, On Opial type inequalities in two independent variables, Proc. Royal Soc. Edinburgh , 100A(1985), 263-270.

[22] B.G. Pachpatte, On certain two dimensional integral inequalities, Chinese J. Math. 17.No. 4 (1989) 273-279.

[23] B.G. Pachpatte, On multidimensional Opial-type inequalities, J. Math. Anal. Appl., 126(1), 85-89, 1987.

[24] B.G. Pachpatte, On some new integral inequalities in ceveral independent variables, Chinese Journal of Mathematics, 14(2), 69-79, 1986.

[25] B.G. Pachpatte, Inequalities of Opial type in three independent variables, Tamkang Journal of Mathematics 35(2), 145-158, 2004.

[26] H. M. Srivastava, K.-L. Tseng, S.-J. Tseng and J.-C. Lo, Some weighted Opial-type inequalities on time scales, Taiwanese J. Math., 14 (2010), 107-122.

[27] Traple, J., On a boundary value problem for systems of ordinary differential equations of second order, Zeszyty Nauk. Univ. Jagiello. Prace Mat. 15 (1971), 159-168.

[28] C.-J. Zhao and W.-S. Cheung, On Opial-type integral inequalities and applications. Math. Inequal. Appl. 17 (2014), no. 1, 223-232.

[29] G. S. Yang. Inequality of Opial-type in two variables. Tamkang J. Math. 13 (1982), 255-259. 